\title{
DNA Sequencing by Electron Microscopy
}

\author{
A.L. Bleloch, C.S. Own, M. Hamalainen, J. Hershleb, K. Kemmish, R. Koene, H. Stark, J. Stark, M. \\ Andregg and W. Andregg \\ Halcyon Molecular, Redwood City, CA94063
}

There are five decades of endeavor behind sequencing by electron microscopy. Slow progress in the last couple of decades belies an early flurry of interest and progress starting at the end of the fifties which then lasted two and a half decades. This period included significant contributions from the work of Albert Crewe. No one said it better than Richard Feynman who clearly marked this early period of progress when he asked "What are the most central and fundamental problems of biology today? They are questions like: What is the sequence of bases in the DNA?... It is very easy to answer many of these fundamental biological questions; you just look at the thing!... Unfortunately, the present microscope sees at a scale which is just a bit too crude." It is significant when an electron microscopist has his or her image in the daily newspaper and Albert Crewe's major contribution was celebrated in many newspapers. (Figure 1 overleaf shows Crewe pointing to an image of single thorium atoms.)

The advent of PCR and sequencing techniques such as the Sanger method had a huge influence on the lack of subsequent progress with this method. Also the ability to visualize DNA albeit not with atomic resolution using scanning probe techniques also contributed to the relative slowing of efforts to image DNA by electron microscopy.

It is generally accepted that it is all but impossible to image a single naked DNA strand with electrons and acquire sequence data before the strand is too damaged to yield useful data. There are notable approaches that attempt to get around this beam damage problem. Amongst them are "diffract and destroy" [2] and graphene supported low-energy TEM imaging techniques [3]. However, both the early studies and the current resurgence of interest use various staining methods to make the DNA sequence visible (see Figures 2 and 3).

Hence the proposed solution to the beam damage problem is to intimately associate by biochemical means a heavy atom label to one of the DNA bases and then utilize the vastly improved contrast in modern microscopes to obtain sequence data. This however, also has its challenges mainly to do with throughput rates. The resurgence in interest comes from the hope for personalised medicine associated with each person having at least their genome sequenced (and perhaps some pathogens, gut flora and possibly a tumor). The magnitude of this problem is significant. There are 6.4million bases which is over $2 \mathrm{~m}$ of double stranded DNA. To image this at atomic resolution would require at least $2 \mathrm{~nm} \times 2 \mathrm{~m}$ or $4 \times 10^{11}$ pixels which is just less than half a terabyte of data. At typical fast imaging speeds in STEM (1kx1k image in $2 \mathrm{~s}$ ), this would take at least 10days of continuous imaging at a coverage of one.

Having posed the problem and covered briefly the history, progress to date will be discussed. (Figure 4 shows an image of labeled DNA obtained on the Oak Ridge UltraSTEM.) It may well turn out that Richard Feynman's prognosis will become more dominant than even he thought probable. 
References

[1] A transcript of Richard Feynman's presentation on December 29th 1959 at the APS annual meeting at Caltech first published February 1960 in Caltech's Engineering and Science. Made available on the web at http://www.zyvex.com/nanotech/feynman.html

[2] R. Neutze, et al., Nature. 406 (2000) 752-757.

[3] R.S.Pantelic, et al, Journal of structural biology, 170 (2010) 152-156

[4] Crewe, et al, Phil. Trans. R. Soc. Lond. B, 261 (1971) 61-7

[5] Cole et al. J Mol Biol 117 (1977) 387-400

[6] The authors would like to thank Steve Pennycook and Andy Lupini for facilitating Fig. 4.

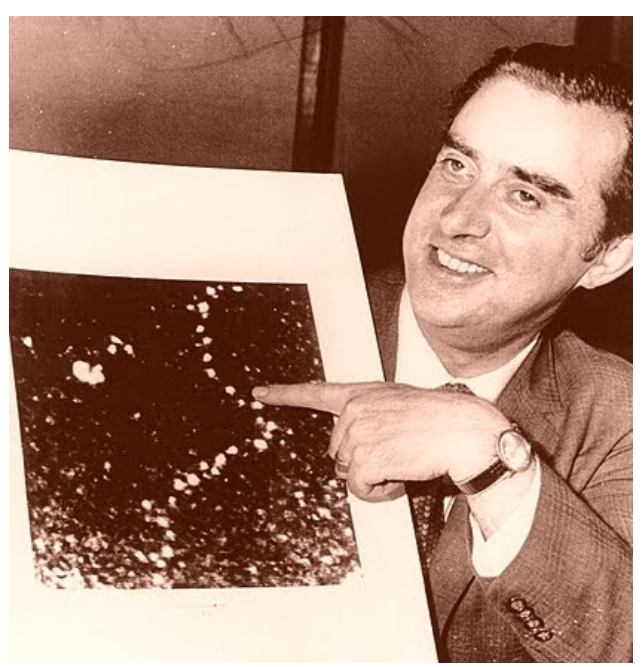

FIG. 1. Albert Crewe in 1970 with a photo of individual thorium atoms

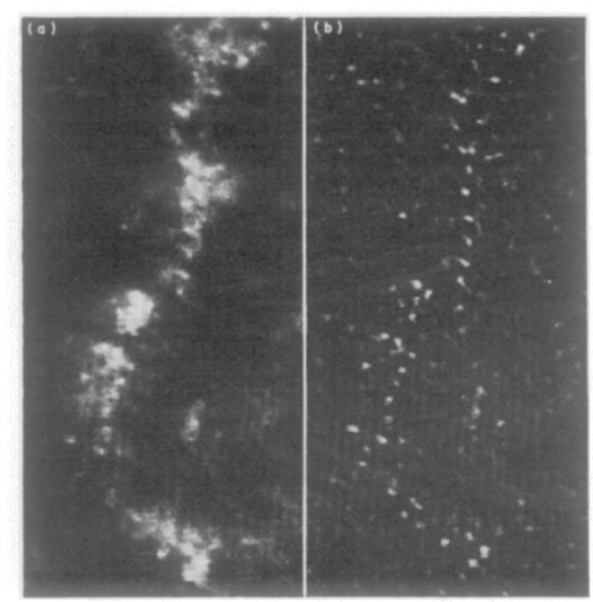

FIG 3 ADF images of strands of DNA labeled with Os atoms from 1977 [5].

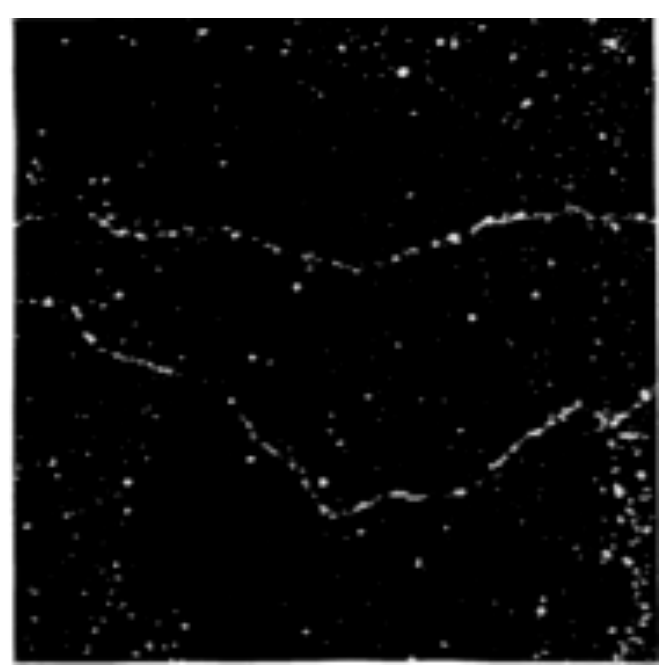

FIG 2. DNA strands stained with Na reproduced from [4]. This is a $\mathrm{Z}$ contrast image (i.e. annular dark field (ADF) divided by elastic brightfield)

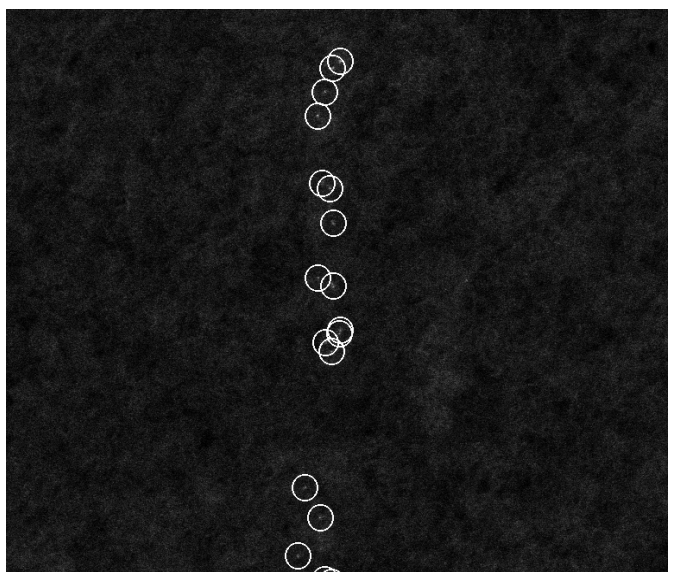

FIG 4. A 20nm part of an image obtained on the UltraSTEM at Oakridge National Laboratories of a strand of DNA labeled with single Os atoms (circled). 